\title{
Acute vasodilator effects of a Rho-kinase inhibitor, fasudil, in patients with severe pulmonary hypertension
}

\author{
Y Fukumoto, T Matoba, A Ito, H Tanaka, T Kishi, S Hayashidani, K Abe, A Takeshita, \\ H Shimokawa
}

$\mathrm{P}$ ulmonary hypertension $(\mathrm{PH})$ is characterised by progressive elevation of pulmonary artery pressure and pulmonary vascular resistance. Pathohistological findings have demonstrated that $\mathrm{PH}$ is associated with abnormal vascular structures, including medial and/or intimal hypertrophy, concentric or eccentric intimal fibrosis, obstruction in the arterial lumen, and aneurysmal dilatation. ${ }^{1}$ Patients with $\mathrm{PH}$ are currently treated with anticoagulant agents, vasodilators including continuous intravenous prostacyclin (prostaglandin $\mathrm{I}_{2}$ ) and oral sildenafil or bosentan, and in end stage, with lung transplantation, ${ }^{1}$ when applicable. Endothelial dysfunction of pulmonary arteries and enhanced pulmonary vasoconstriction contribute to the development of $\mathrm{PH} .{ }^{1}$ Endothelial nitric oxide synthase (eNOS) expression is reduced in patients with $\mathrm{PH}$ and therefore nitric oxide inhalation and sildenafil are useful for those patients. However, more effective treatments remain to be developed. ${ }^{1}$

We have recently demonstrated that Rho-kinase is substantially involved in the pathogenesis of a wide range of cardiovascular disease. ${ }^{23}$ Rho-kinase suppresses myosin phosphatase activity by phosphorylating the myosin binding subunit of the enzyme and thus augments vascular smooth muscle cell (VSMC) contraction at a given intracellular calcium concentration. ${ }^{4}$ Indeed, we have demonstrated that a Rho-kinase inhibitor, fasudil, suppresses abnormal hyperconstriction of forearm and coronary arteries in animals and humans. ${ }^{2}{ }^{3}$ Moreover, we have recently demonstrated that Rho-kinase inhibition increases eNOS expression and decreases inflammatory cell migration or anigiotensin II induced mRNA expression of monocyte chemoattractant protein- 1 and plasminogen activator inhibitor- 1 in vivo and in vitro. ${ }^{3}$ Our in vivo study also indicated that long term oral treatment with fasudil notably ameliorates monocrotaline induced $\mathrm{PH}$ and pulmonary vascular lesions in rats. ${ }^{5}$ In the present study, we thus examined the acute vasodilatory effects of pulmonary circulation by intravenous administration of fasudil in patients with severe $\mathrm{PH}$.

\section{METHODS}

The ethical committee of the Kyushu University Hospital approved this study and all patients provided informed consent before the study. Nine patients with severe $\mathrm{PH}$ (mean age 53 years; range 26-76 years, three men and six women) were prospectively enrolled to examine the acute effects of a Rho-kinase inhibitor, fasudil hydrochloride, on pulmonary circulation at the Kyushu University Hospital. All patients underwent right heart cardiac catheterisation while they continued all medications, including oral or intravenous prostaglandin $\mathrm{I}_{2}$. We measured pulmonary vascular resistance (PVR), systemic and pulmonary arterial blood pressure (PAP), and cardiac index, using a Swan-Ganz catheter. We examined the response to intravenous administration of fasudil hydrochloride (30 mg for 30 minutes).

Blood samples were taken before and 1, 2, 4, and 8 hours after intravenous administration of fasudil hydrochloride to measure serum concentrations of fasudil and hydroxyfasudil, an active metabolite of fasudil (Daiichi Chemical and Pharmacological Company, Ibaragi, Japan). ${ }^{2}$

Abbreviations: eNOS, endothelial nitric oxide synthase; $C_{\max }$ maximum serum concentration; PAP, pulmonary arterial blood pressure; $\mathrm{PH}$, pulmonary hypertension; PVR, pulmonary vascular resistance; VSMC, vascular smooth muscle cell
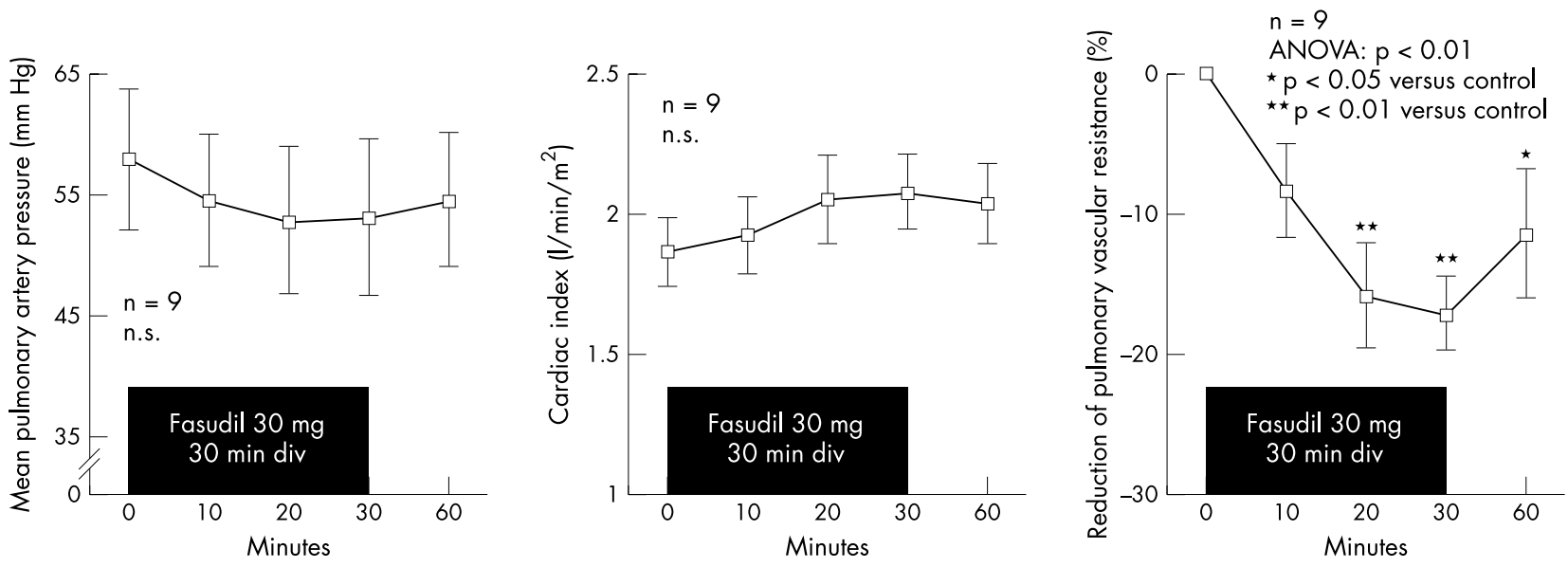

Figure 1 Intravenous administration of fasudil slightly decreased pulmonary artery pressure (left panel) and increased cardiac index (middle panel), although there were no significant differences. However, fasudil significantly reduced pulmonary vascular resistance (right panel). Data are expressed as mean (SD) and analysed by ANOVA followed by a Fisher's post hoc test. 


\section{RESULTS}

Mean systolic/diastolic/PAP at baseline was 94/36/58 mm Hg and mean PVR index was 2429 dyne* $\mathrm{sec}^{*} \mathrm{~cm}^{-5 *} \mathrm{~m}^{2}$. Figure 1 shows that intravenous administration of fasudil slightly decreased PAP and increased cardiac index in these patients, although there were no significant differences; however, it significantly reduced PVR by $17 \%$ in nine patients. There were no side effects, including systemic hypotension.

Intravenous administration of fasudil achieved maximum serum concentration $\left(C_{\max }\right)$ of 278.9 (150.5) $\mathrm{ng} / \mathrm{ml}$ of fasudil and $173.2(10.3) \mathrm{ng} / \mathrm{ml}$ of hydroxyfasudil. Time to achieve half of $\mathrm{C}_{\max }\left(\mathrm{T}^{1 / 2}\right)$ was $0.5(0.6)$ hours for fasudil and 3.6 (1.6) hours for hydroxyfasudil.

\section{DISCUSSION}

Based on our findings in animals, we examined the possible involvement of Rho-kinase in the pathogenesis of $\mathrm{PH}$ in humans in the present study and the results indicated that it is indeed involved. Increased PVR may be caused by both pulmonary vascular remodelling and sustained pulmonary vascular vasoconstriction. ${ }^{1}$ The mechanisms involved in the latter include endothelial dysfunction and VSMC hyperconstriction. ${ }^{1}$ In the present study, fasudil reduced PVR, indicating that VSMC hyperconstriction in $\mathrm{PH}$ is partially caused by increased Rho-kinase activity. Our series of experiments suggest that long term blockade of Rho-kinase causes a regression of vascular remodelling, ${ }^{35}$ although the $\mathrm{C}_{\max }$ of fasudil/hydroxyfasudil in the present study indicates its vasodilatory effect but not its antiproliferative one. ${ }^{3}$ We plan to examine whether additional long term oral treatment with fasudil further decreases PAP and improves quality of life and survival of patients with severe $\mathrm{PH}$.

\section{ACKNOWLEDGEMENTS}

This study was supported in part by the grants-in-aid (numbers 13307024, 13557068) and the grant for the 21st Century COE Program from the Japanese Ministry of Education, Culture, Sports, Science and Technology, Tokyo, Japan and from the Program for Promotion of Fundamental Studies in Health Sciences of the Organization for Pharmaceutical Safety and Research of Japan.

\section{Authors' affiliations}

Y Fukumoto, T Matoba, A Ito, H Tanaka, T Kishi, S Hayashidani, K Abe, A Takeshita, H Shimokawa, Department of Cardiovascular Medicine, Kyushu University Graduate School of Medical Sciences, Fukuoka, Japan

Correspondence to: Dr Yoshihiro Fukumoto, 3-1-1 Maidashi, Higashiku, Fukuoka 812-8582, Japan; yoshif@cardiol.med.kyushu-u.ac.jp

Accepted 14 May 2004

\section{REFERENCES}

1 Runo JR, Loyd JE. Primary pulmonary hypertension. Lancet 2003:361:1533-44.

2 Masumoto A, Hirooka Y, Shimokawa H, et al. Possible involvement of Rhokinase in the pathogenesis of hypertension in humans. Hypertension 2001;38:1307-10.

3 Shimokawa H. Rho-kinase as a novel therapeutic target in treatment of cardiovascular disease. J Cardiovasc Pharmacol 2002;39:319-27

4 Somlyo AP, Somlyo AV. Signal transduction by G-proteins, Rho-kinase and protein phosphatase to smooth muscle and non-muscle myosin II. J Physiol 2000;522:177-85

5 Abe K, Shimokawa H, Morikawa K, et al. Long-term treatment with a Rhokinase inhibitor improves monocrotaline-induced fatal pulmonary hypertension in rats. Circ Res 2004;94:385-93.

\section{IMAGES IN CARDIOLOGY} Multislice CT of the abdominal aorta in a patient with Takayasu's arteritis reveals malformation of collateral arteries and occlusion of abdominal aorta

A 57 year old woman with Takayasu's arteritis presented with abdominal discomfort. A previous enhanced computed tomography (CT) had revealed occlusion of mid portion of the abdominal aorta, but both femoral arteries had a pulse.

To evaluate the whole aorta, enhanced multislice CT (Light Speed Ultra 16, General Electric, Milwaukee, Wisconsin, USA) was performed after injection of contrast with retrospective ECG gated reconstruction with a $1.25 \mathrm{~mm}$ slice thickness (helical pitch 6.00) covering the cervical and thoracic areas, and non-ECG gated acquisition with a $1.25 \mathrm{~mm}$ slice thickness (helical pitch 13.75), that covered the abdomen and upper portion of the lower limbs. Data were transferred to a workstation (Virtual Place, Office Azemoto, Tokyo, Japan).

Axial source and coronal view of multiplanar reconstruction images revealed occlusion of the abdominal aorta with advanced calcification. Volume rendering images of the thorax revealed no abnormal findings except enlarged left and right internal thoracic arteries (LITA and RITA, respectively). The image of the abdomen and proximal portion of lower limbs revealed abnormally enlarged collateral arteries originating from enlarged inferior epigastric arteries (IEA), which continued from enlarged LITA and RITA, and deep iliac circumflex arteries (DICA). IEA and DICA fed into the distal portion of both common iliac arteries (CIA).



\section{N Funabashi \\ M Asano \\ I Komuro}

nobusada@ma.kcom.ne.jp 